\title{
VIDAS ARRISCADAS: UMA REFLEXÃO SOBRE A RELAÇÃO ENTRE O NÚMERO DE GESTAÇÕES E MORTALIDADE MATERNA
}

\section{ENDANGHED LIVES: A REFLEXION ABOUT THE RELATION BETWEEN GESTATIONS NUMBER AND MATERNAL MORTALITY}

Vânia Muniz Néquer Soares *

Néia Schor **

Carlos Mendes Tavares ***

Vidas Arriscadas: Uma Reflexão Sobre a Relação Entre o Número de Gestações e Mortalidade Materna. Rev Bras Crescimento Desenvolvimento Hum. 2008; 18(3): 254-263

\section{Resumo:}

Este estudo teve por objetivo identificar o risco e os fatores associados à mortalidade materna de multigestas (cinco ou mais gestações). Descreve a tendência da Taxa de Fecundidade Total (TFT) e da Razão de Mortalidade Materna (RMM) de 1998 a 2004 no Paraná. Apresenta uma análise dos 822 óbitos maternos deste período, o cálculo da Razão de Mortalidade Materna Específica, o Risco Relativo, a freqüência e o Odds Ratio para algumas variáveis segundo número de gestações. Analisa também as causas e a evitabilidade dos óbitos maternos. Os resultados apontaram TFT baixa (1,8 filhos por mulher) e a RMM elevada (69,7/100.000 nascidos vivos) no Paraná em 2004. Um quarto dos 822 óbitos maternos (206) era de multigestas (cinco ou mais gestações), o risco relativo de morte materna foi seis vezes superior do que para as mulheres com até duas gestações, e os perfis sociodemográfico e o reprodutivo foram mais desfavoráveis para aquele grupo de mulheres. A baixa escolaridade, a idade igual ou acima de 30 anos e o pré-natal com menos de quatro consultas apresentaram associação com o maior número de gestações. A proporção de mortes por causas obstétricas indiretas, hemorragias e aborto, foi maior entre as multigestas, e cerca de 90\% dos óbitos deste grupo foi considerado evitável. Concluiu-se que o monitoramento das multigestas com idade avançada e em desvantagem social, bem como ações efetivas de planejamento familiar, e serviços obstétricos qualificados para gestação de alto risco são medidas que podem contribuir para a redução da mortalidade materna.

Palavras-chave: Multigesta; multiparidade; mortalidade materna; risco reprodutivo

\footnotetext{
* $\quad$ Doutora em Saúde Pública. Enfermeira Sanitarista da Secretaria de Estado da Saúde do Paraná.

** Professor Titular. Departamento de Saúde Materno-infantil Faculdade de saúde Pública/USP

*** Estatístico. Mestre em Estudos Populacionais. Doutor em Saúde Pública. Instituto Nacional de Estatística. Cabo Verde, África.

Correspondência para: Néia Schor. Departamento de Saúde Materno Infantil da Faculdade de Saúde Pública da Universidade de São Paulo. E-mail: shor@usp.br
} 


\begin{abstract}
:
This study had as objective to identify the risk and the factors associated to the multigesta's maternal mortality (gesta $\geqslant 5$ ). It describes the tendency of the Total Fertility Rate (TFT) and of the Maternal Mortality Ratio (RMM) between 1998 to 2004 in Paraná. It presents an 822 maternal deaths analysis in this period, the calculation of the Specific Maternal Mortality Ratio, the Relative Risk, the frequency and the Odds Ratio for some variables, according to number of gestations. Analyzes also the causes and the prevent way the maternal deaths. The results aimed low TFT (1.8 children by woman) and to RMM elevated (69,7/100.000 been born alive) in Paraná in 2004. A quarter of the 822 maternal deaths (206) was of multigestas (gesta $\geqslant 5$ ), the maternal death relative risk was six times higher than for the women with to two gestations, and the profiles sociodemographic and the reproductive one were more unfavorable for that group of women. Incomplete schooling, equal age or above 30 years and less than four prenatal medical consultations presented association with the most greatest number of gestations. The deaths proportion by indirect obstetric causes, hemorrhages and abortion was bigger between the multigestas and around $90 \%$ of the deaths of this group was considered avoidable. It concluded that monitoring of the multigestas with advanced age and in social disadvantage, as well as effective actions of family planning, and obstetric service qualified for high risk gestation are measures that can contribute for reduction of the maternal mortality in Paraná.
\end{abstract}

Key words: Pregnancy; multiparity; maternal mortality; reproductive risk.

\section{INTRODUÇÃO}

Reduzir a mortalidade materna não é só um direito humano, mas um imperativo de saúde pública reafirmam os organismos internacionais. Nesta perspectiva a Organização das Nações Unidas - ONU, durante a Cúpula do Milênio da ONU em 2000 adotou como um dos oito Objetivos de Desenvolvimento do Milênio (ODM), "Melhorar a saúde materna”, tendo como meta inicial, a redução em três quartos, da Razão de Mortalidade Materna (RMM) - número de mortes maternas em 100.000 nascidos vivos - entre 1990 e $2015 .^{1}$

RMM dos países pobres estimada, para o ano de 2005, em 450 mortes por 100.000 nascidos vivos, contrasta acentuadamente com a RMM das regiões desenvolvidas, estimada em 9 mortes maternas por 100.000 nascidos vivos. Para a América Latina e Caribe com cerca de 15.000 mortes maternas anuais, calculou-se uma RMM de 130 óbitos por 100.000 nascidos vivos (NV). ${ }^{1}$
Outro indicador, o Risco de Morte Materna Feminino (RMMF) que representa o risco de morte materna ao longo da vida de uma mulher, e que é calculado pela relação entre o número de óbitos maternos e o número de mulheres, foi estimado em 1 para cada 290 mulheres na América Latina, contra 1 em 7300 nos países desenvolvidos. Estes indicadores revelam o risco de morte para uma mulher em cada gravidez. Considera-se, portanto, que, em cenários de alta fecundidade, cada mulher enfrentaria este risco muitas vezes.

O elevado número de gestações e partos seria, desta forma, fator de risco para morte materna. Entretanto a literatura consultada mostra que existem controvérsias a respeito do risco de morte relacionado à paridade. Alguns autores consideram a baixa primiparidade como de maior risco, outros a multiparidade, e ainda outros não atribuem efeitos que, por si só, expliquem o aumento do risco de morte materna pelo aumento da paridade..$^{2-5}$ 
Estudo americano publicado em 2005 e que realizou ampla revisão da literatura sobre este tema, refere como incerta a associação entre multiparidade (número de partos $\geqslant 5$ ) e resultados adversos na gestação. Segundo os autores, os achados que associam alta paridade e resultados maternos e fetais não são consistentes; enquanto a literatura mais antiga tende a sugerir que multiparidade é um fator de risco negativo para o nascimento, a literatura mais recente não sustenta esses achados. ${ }^{5}$

Alguns efeitos adversos apontados na literatura relacionados à alta paridade incluem a má apresentação fetal, descolamento prematuro de placenta, trabalho de parto disfuncional e hemorragia pós-parto. No entanto, alguns estudos concluem que estes efeitos desfavoráveis estariam relacionados à idade avançada na gestação e/ou às condições socioeconômicas mais desvantajosas apresentadas normalmente pelas mulheres com alta paridade. ${ }^{2-5}$

A oferta de serviços de planejamento familiar é destacada neste sentido como estratégica para a redução da mortalidade materna, contribuindo inicialmente para reduzir o número absoluto de gestações, para aumentar o intervalo entre as gestações e evitar as gestações nas faixas etárias de maior risco. No entanto, já se evidenciou em muitos países que apenas o processo de controle da fecundidade apresenta impacto limitado na redução da mortalidade materna. A manutenção de elevadas taxas de mortalidade materna, em países e regiões onde a fecundidade já decresceu, estaria mais fortemente relacionada às dificuldades de acesso aos cuidados obstétricos essenciais, ao tratamento das emergências obstétricas, ao acompanhamento das gestações de maior risco e também à pobreza e às desigualdades de gênero. ${ }^{6}$

A acelerada queda da fecundidade observada em nosso país não vem sendo acompanhada por uma queda expressiva da mortalidade materna. Segundo a PNDS (Pesquisa Nacional de Demografia e Saúde) $2006^{7}$, o Brasil alcançou uma taxa de fecundidade de 1,8 filhos por mulher em 2006, índice que está abaixo do chamado nível de reposição populacional, mas a RMM estimada permanece estável em altos níveis, próxima a 100 óbitos por 100.000 nascidos vivos, ficando evidente que apenas o controle da fecundidade não tem sido suficiente para reduzir as mortes maternas também no Brasil. ${ }^{8,9}$

Documento da Organização Pan-americana de Saúde (OPAS) reforça esta constatação ao analisar a situação da mortalidade materna na América Latina e Caribe ao comentar que (...) "em comparação com outros indicadores, a mortalidade materna é um problema no qual pouco progresso foi alcançado. Embora muitos indicadores de saúde como a taxa de fecundidade e de natalidade tenham melhorado muito durante as duas últimas décadas, as taxas de mortalidade materna permaneceram estagnadas. As causas desta situação estão enraizadas na inadequação das várias intervenções que visam a saúde materna, assim como nas iniqüidades em função do gênero". ${ }^{10}$

O detalhamento dos possíveis determinantes de cada óbito materno é, portanto, essencial para o enfrentamento deste problema de saúde que afeta não só as mulheres, mas a sociedade em geral.

A partir destas reflexões acerca da constatação da ocorrência, ainda relativamente freqüente, de óbitos maternos de mulheres com cinco ou mais gestações, baseada nos dados dos Comitês de Prevenção da Mortalidade Materna do Paraná, realizamos este estudo com o objetivo de identificar o risco e os fatores associados à mortalidade materna de multigestas (cinco ou mais gestações) no Paraná.

\section{MÉTODO}

Trata-se de um estudo descritivo, de corte transversal, em que foram analisados 822 
óbitos maternos ocorridos no período de 1998 a 2004 (período em que os dados estavam disponíveis) no Estado do Paraná, obtidos no banco de dados do Comitê Estadual de Prevenção da Mortalidade Materna (CEPMM).

Os comitês atuam no Paraná desde 1989, analisando todos os óbitos maternos, identificados e investigados pelas equipes de vigilância epidemiológica dos municípios, utilizando o método RAMM (Reproductive Age Maternal Mortality), corrigindo a subnotificação dos óbitos maternos, e dando confiabilidade aos dados do estado. ${ }^{11}$

Os dados da ficha de investigação, dos óbitos maternos, são digitados pelo CEPMM e organizados em banco de dados Access, o qual foi disponibilizado para este estudo. São, portanto, dados secundários, não sendo fornecidos dados como nome das mulheres e dos serviços onde ocorreram os óbitos, preservando-se o anonimato dos mesmos.

Foram calculadas, inicialmente, a RMM e a Taxa de Fecundidade Total (TFT) do período 1998 a 2004 demonstrando a evolução destas taxas no Estado.

A taxa de fecundidade total (TFT) refere-se ao número médio de filhos nascidos vivos tidos por uma mulher, ao final do seu período reprodutivo, inserida na população residente em determinado espaço geográfico, no ano considerado. Foi calculada diretamente pelo somatório das taxas específicas de fecundidade para as mulheres de 15 a 49 anos e multiplicado por cinco. O número de nascidos vivos foi obtido no Sistema de Informações sobre Nascidos Vivos (SINASC/MS), e o da população feminina por faixa etária foi obtido junto ao DATASUS/MS. ${ }^{12}$

A RMM foi calculada pela relação entre o número de óbitos por complicações de gravidez, parto e puerpério até 42 dias pós-parto, e o número de nascidos vivos (NV), expresso por 100.000 NV. Não foram incluídos os óbitos maternos tardios, que correspondem aos ocorridos após os 42 dias de puerpério até um ano pós-parto. ${ }^{12}$

Posteriormente foi desenvolvido o cálculo da RMM específica (RMME) segundo o número de gestações, para dois subgrupos, um abrangendo os óbitos maternos de mulheres com até duas gestações (primigestas e secundigestas), e o outro abrangendo os óbitos das mulheres com cinco ou mais gestações (aqui denominadas multigestas), calculando-se a partir destas taxas o risco relativo para os dois grupos das mulheres, buscando comparar os fatores associados e os riscos para morte materna entre as mulheres com pequeno número de gestações e as multigestas.

As variáveis independentes selecionadas para o estudo foram: idade, escolaridade, número de consultas de pré-natal e tipo de parto, além da causa e da evitabilidade do óbito. A variável dependente foi o número de gestações. Calculou-se o odds ratio para medir a associação entre as variáveis.

\section{RESULTADOS}

De acordo com a Tabela 1, constatou-se uma queda de 14,0\% na Razão da Mortalidade Materna entre 1998 e 2004. No entanto, a RMM de 69,7 por 100.000 nascidos vivos em 2004, indica que o Paraná apresenta ainda elevada mortalidade materna. A taxa de fecundidade total também apresentou uma acentuada queda, $18 \%$ em seis anos, reduzindo de 2,2 filhos por mulher em 1998 para 1,8 em 2004.

Na tabela 2, a RMM específica das mulheres multigestas (com cinco ou mais gestações) do período 1998 - 2004 foi de 278,33 por 100.000 nascidos vivos e a de mulheres com até duas gestações foi 48,1 por 100.000 nascidos vivos, demonstrando um risco reprodutivo seis vezes maior para as grande multigestas.

De acordo com a tabela 3, 80,2\% das mulheres multigestas tinham 30 anos ou mais 
Tabela 1: Número de óbitos maternos. Número de nascidos vivos. Razão de Mortalidade Materna (RMM) e Taxa de Fecundidade Total (TFT), Paraná, 1998 - 2004

\begin{tabular}{lcccc}
\hline ANO & $\begin{array}{c}\text { Número de óbitos } \\
\text { maternos* }\end{array}$ & $\begin{array}{c}\text { Número de nascidos } \\
\text { vivos** }\end{array}$ & RMM & TFT*** \\
\hline 1998 & 150 & 185.097 & 81,04 & 2,2 \\
1999 & 147 & 186.111 & 78,99 & 2,2 \\
2000 & 119 & 179.160 & 66,42 & 2,1 \\
2001 & 109 & 166.990 & 65,27 & 1,9 \\
2002 & 95 & 164.812 & 57,64 & 1,9 \\
2003 & 91 & 156.997 & 57,96 & 1,8 \\
2004 & 111 & 159.133 & 69,70 & $-18,2 \%$ \\
Variação (98-04) & 822 & 1.198 .435 & $-14,0 \%$ & \\
\hline
\end{tabular}

Obs: dados obtidos * Comitê Estadual de Prevenção da Mortalidade Materna-CEPMM/Secretaria Estadual de Saúde do Paraná_SESA, **SINASC/MS, tabela elaborada pelos autores

Tabela 2: Número de óbitos maternos. Número de nascidos vivos.Razão de Mortalidade Materna (RMM) e Risco Relativo (RR) segundo número de gestações. Paraná, 1998 - 2004.

\begin{tabular}{llcccc}
\hline \multicolumn{1}{c}{$\mathbf{N}^{\mathbf{0}}$ gestações } & $\begin{array}{c}\mathbf{N}^{\mathbf{0}} \text { óbitos } \\
\text { maternos* }\end{array}$ & \% óbitos maternos & $\begin{array}{c}\text { Número de } \\
\text { nascidos vivos** }\end{array}$ & RMM & RR \\
\hline Até duas & 402 & $48,9 \%$ & 835.832 & 48,1 & 1 \\
Cinco ou mais & 206 & $25,1 \%$ & 74.013 & 278,3 & 6 \\
Total & $822^{* * *}$ & 100 & 1.198 .435 & 68,59 & - \\
\hline
\end{tabular}

Obs: dados obtidos * Comitê Estadual de Prevenção da Mortalidade Materna-CEPMM/Secretaria Estadual de Saúde do Paraná_SESA, **SINASC/MS, tabela elaborada pelos autores.

de idade e $78,3 \%$ baixa escolaridade (menos de 8 anos de estudo), sendo essas proporções bastante inferiores para as mulheres com até duas gestações, 22,9\% e 54,7\% respectivamente; apresentaram ainda maior proporção de prénatal incompleto, 37\% contra 19\% das com até duas gestações, e 50,7\% foram submetidas a partos cesáreos contra 64\% das mulheres com até duas gestações.

Evidenciou-se ainda que os fatores associados positivamente com o elevado número de gestações foram: a idade maior ou igual a 30 anos, escolaridade menor que 8 anos e o número de consultas de pré-natal menor que 4. A taxa de cesáreas não apresentou associação com número de gestações.

Analisando as causas dos óbitos maternos (Tabela 4) observa-se que as multigestas (cinco ou mais gestações) apresentaram maior proporção de óbitos por causas obstétricas in- diretas (principalmente as doenças do aparelho cardiocirculatório e respiratório complicando a gestação), óbitos por hemorragias ante e pós-parto e óbitos por aborto em relação às mulheres com até duas gestações. Foram considerados evitáveis cerca de $90 \%$ dos óbitos maternos das multigestas.

\section{DISCUSSÃO}

O Paraná, como a maior parte dos estados brasileiros, já apresenta taxas de fecundidade abaixo do nível de reposição populacional, 2,1 filhos por mulher., ${ }^{7,12}$ No entanto, a RMM permanece elevada, de 69,7 por 100.000 nascidos vivos, sendo quase oito vezes superior à taxa dos países desenvolvidos (9 por 100000 nascidos vivos). ${ }^{1}$ 
Tabela 3: Número, percentual e odds ratio (OR) dos óbitos maternos segundo número de gestações e variáveis sociodemográficas e reprodutivas. Paraná, 1998 -2004

\begin{tabular}{lccc}
\hline Variáveis & \multicolumn{2}{c}{$\mathrm{N}^{\text {0 }}$ de gestações $(\%)$} & OR \\
& Até duas & Cinco ou mais & \\
\hline Totais & 206 & 402 & \\
Idade: $\geqslant 30$ anos & $166(80,2)$ & $92(22,9)$ & 13,6 \\
$<30$ anos & $41(9,8)$ & $309(77,1)$ & \\
Escolaridade: $\leqslant 8$ anos & $126(78,3)$ & $187(54,7)$ & 2,98 \\
$\quad>8$ anos & $35(21,7)$ & $155(45,3)$ & \\
N. de consultas de pré natal & & $60(19,7)$ & 2,43 \\
$<4$ & $50(37,3)$ & $245(88,3)$ & 0,58 \\
$>4$ & $84(62,7)$ & $190(64,0)$ & \\
Parto: Cesárea & $76(50,7)$ & $107(36,0)$ & \\
Normal & $74(49,3)$ &
\end{tabular}

Obs.: dados obtidos do CEPMM/SESA- tabela elaborada pelos autores. As diferenças em relação aos subtotais de cada variável deve-se à exclusão dos ignorados. O total de óbitos maternos no Paraná no período estudado foi de 822 óbitos.

Tabela 4: Número e percentual dos óbitos maternos segundo causa básica, evitabilidade dos óbitos maternos e número de gestações. Paraná, 1998 -2004

\begin{tabular}{|c|c|c|c|c|}
\hline \multirow{3}{*}{ Causas } & \multicolumn{4}{|c|}{ Número de gestações } \\
\hline & \multicolumn{2}{|c|}{ Até duas } & \multicolumn{2}{|c|}{ Cinco ou mais } \\
\hline & $\mathrm{N}^{\mathrm{o}}$ & $\%$ & $\mathrm{~N}^{\circ}$ & $\%$ \\
\hline \multicolumn{5}{|l|}{ Obstétricas diretas } \\
\hline Aborto & 17 & 8,2 & 18 & 4,5 \\
\hline DHGE & 32 & 15,5 & 86 & 21,4 \\
\hline Hemorragia anteparto & 15 & 7,3 & 5 & 1,2 \\
\hline Hemorragia pós-parto & 27 & 13,1 & 34 & 8,5 \\
\hline Infecção puerperal & 8 & 3,9 & 44 & $10, \mathrm{c}$ \\
\hline Infecção geniturinária & 5 & 2,4 & 30 & 7,5 \\
\hline Embolia & 11 & 5,3 & 27 & 6,7 \\
\hline Complicações anestésicas & 4 & 1,9 & 16 & 4,0 \\
\hline Outras & 19 & 9,2 & 23 & 5,7 \\
\hline Obstétricas indiretas & 53 & 25,7 & 88 & 21,9 \\
\hline Causa desconhecida & 15 & 7,3 & 31 & 7,7 \\
\hline \multicolumn{5}{|l|}{ Evitabilidade } \\
\hline Sim & 179 & 87,0 & 326 & 81,1 \\
\hline Não & 15 & 7,3 & 38 & 9,4 \\
\hline Inconclusivo & 12 & 5,8 & 38 & 9,4 \\
\hline Total & 206 & 100 & 402 & 100 \\
\hline
\end{tabular}

Obs.: Dados obtidos do CEPMM/SESA- tabela elaborada pelos autores

As diferenças em relação aos subtotais de cada variável deve-se à exclusão dos ignorados. O total de óbitos maternos no Paraná no período estudado foi de 822 óbitos.

Demonstrou-se, por outro lado, que é necessária certa cautela em relação ao declínio da fecundidade, pois as médias estimadas muitas vezes ocultam contrastes destas taxas, podendo haver variações no padrão de fecundidade de segmentos específicos da população e em diferentes regiões.

Este estudo evidenciou que existe ainda um grupo de mulheres com elevado número de gestações e com alto risco para mortali- 
dade materna no Paraná, e que as condições sociodemográficas e reprodutivas destas mulheres eram mais desfavoráveis, o que pode contribuir para elevação deste risco, além das falhas identificadas na assistência.

A baixa escolaridade das mulheres estudadas pode ter interferido nas possibilidades destas em obter e utilizar as informações sobre contracepção e saúde, reduzindo suas oportunidades de inserção no mercado de trabalho, e de obtenção de melhor renda, como demonstram diversos estudos. A garantia de maior escolaridade facilitaria o acesso e utilização de métodos contraceptivos mais adequados, contribuindo para a redução das gestações indesejadas, e dos riscos de morte materna. $^{13,14}$

A idade acima de 30 anos foi identificada como um fator fortemente associado à mortalidade materna para as multigestas. Muitos estudos referem que a idade interfere na mortalidade materna; as mulheres acima de 35 anos são consideradas como de alto risco, porém alguns autores ressaltam que a idade deve ser associada a outros fatores como as condições socioeconômicas e qualidade dos serviços para gestação de risco. ${ }^{2-5,15,16}$

Estudo realizado por LANG e KING (2008) nos Estados Unidos identificou pequenos progressos na redução da RMM desde 1982 no país. Explicações para a estagnação das taxas, segundo os autores, incluem as gestações mais tardias, o potencial de complicações associadas à idade reprodutiva avançada (particularmente acima de 35 anos) e a multiparidade. Observaram crescimento das gestações multifetais, devido à reprodução assistida e o aumento de casos de óbito por miocardiopatias periparto. As principais causas de mortalidade materna nos Estados Unidos são tromboembolismo, hemorragias, hipertensão e suas complicações, e infecção. ${ }^{15}$

Na Argentina, Ramos et al. ${ }^{16}$ realizaram uma ampla avaliação da mortalidade materna com o objetivo de fortalecer o sistema de vigi- lância das mortes maternas e reorientar as políticas de saúde reprodutiva, em cooperação com a Organização Mundial de Saúde (OMS) desenvolveram estudo multicêntrico de base populacional, abrangendo seis estados com maior RMM. Identificaram como principais causas de mortalidade materna o aborto, seguido das hemorragias, infecções/septicemia e transtornos hipertensivos. Dentre os fatores de risco, a ausência da atenção obstétrica essencial, a atenção ativa nas emergências obstétricas, a idade acima de 35 anos e a multiparidade contribuíram para elevar o risco de morte materna. Outros fatores que contribuíram para as mortes maternas na Argentina foram o atraso no reconhecimento dos sinais de alarme, a tentativa de ocultamento do aborto, retardamento do tratamento devido a diagnósticos errôneos, demora na transferência dos casos de risco, falta de recursos e de transporte. A RMM na Argentina em 2002 era de 46/100.000 nascidos vivos, porém com diferenças entre as regiões. A RMM em Buenos Aires era de 14/ 100.000 nascidos vivos, enquanto que em Formosa, era de 166/100.000 nascidos vivos, quase 12 vezes maior. A subnotificação da mortalidade materna estimada pelo estudo foi de apenas $9,5 \%{ }^{16}$

Estudo sobre mortalidade materna realizado no Recife por Costa ${ }^{17}$, observou uma RMM quatro vezes superior na faixa etária a partir dos 35 anos (RMM de 237/100.000 nascidos vivos), em relação à faixa de 20 a 34 anos (RMM de 65/100.000 nascidos vivos) o que reforça que idade constitui um fator de risco para morte materna. ${ }^{17}$

Com relação às causas dos óbitos, identificou-se que as causas obstétricas indiretas foram a primeira causa de morte materna para as multigestas (cinco ou mais gestações). Essas são observadas na literatura como causas associadas às gestantes com idade mais avançada, portadoras de doenças crônicas caracterizando, nestas condições, gestação de alto risco e reforçando a necessidade de 
acompanhamento das mesmas em serviço especializado. No entanto, o que se evidenciou foi um menor número de consultas pré-natal realizadas por essas mulheres.

Resultado semelhante foi identificado em estudo realizado no município de Caxias do Sul, no estado do Rio Grande do Sul, onde se verificou que quanto maior o número de gestações, mais tardiamente a gestante iniciou o pré-natal, realizando um menor número de consultas. Os motivos alegados para baixa participação nas consultas foram o fato de o médico não examiná-las, a dificuldade de marcar consultas e a falta de informação a respeito da importância do pré-natal, demonstrando a baixa qualidade dos serviços percebida pelas

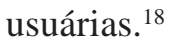

As hemorragias anteparto e pós-parto, em conjunto, constituíram a segunda causa de morte das mulheres grandes multigestas, com uma proporção superior aos casos de Doença Hipertensiva Específica da Gravidez (DHEG), que constitui a primeira causa de morte materna no Paraná. A literatura refere que as hemorragias são complicações freqüentes entre as

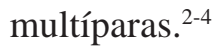

Os óbitos maternos devido ao aborto, com proporção duas vezes superior à das mulheres com até duas gestações, poderiam ter sido evitados em parte pela inclusão, em programas de planejamento familiar mais efetivos, das mulheres que não desejavam mais engravidar, atendendo a suas necessidades particulares e identificando as dificuldades na utilização e na adaptação aos métodos contraceptivos. A garantia ao aborto seguro e ao tratamento adequado das suas complicações também se mostra indispensável. ${ }^{6}$

Estudos apontam que é provável que ainda existam na população dois segmentos de mulheres: um que está em contato direto com os serviços formais de saúde, freqüentando o pré-natal e o serviço de planejamento familiar, e que tem, portanto, menores riscos, e outro segmento que não tem acesso ou tem maior dificuldade de frequentar esses serviços e teriam maior risco reprodutivo. Os programas tradicionais de planejamento familiar podem estar excluindo mulheres que têm alto risco intrínseco, que são aquelas que não procuram os serviços e que não estariam sendo identificadas pelos mesmos. A inclusão estratégica desse grupo de mulheres em serviços de saúde reprodutiva qualificados poderia impactar na mortalidade materna. ${ }^{6}$

A ampliação do acesso no SUS, a atenção integral à saúde da mulher incluindo o planejamento familiar e o pré-natal qualificados como prevê o pacto de atenção básica é essencial para a redução da mortalidade materna.

Verificou-se ainda que tanto o grupo de mulheres com até duas gestações como o grupo multigestas (5 ou mais gestações) apresentaram altas taxas de cesáreas. A literatura e as evidências apontam que as altas taxas de cesáreas podem determinar com maior freqüência resultados adversos do parto, dentre eles hemorragias,que neste estudo foi a segunda causa de morte das multigestas.

Um estudo da Organização Mundial da Saúde (OMS), coordenado por Villar ${ }^{19}$ realizado em 120 hospitais públicos e privados em oito países da América Latina, dentre eles Argentina, Brasil, Cuba, Equador, México, Nicarágua, Paraguai e Peru, identificou que um terço dos 97 mil partos analisados durante o período do estudo foi realizado por cesariana; evidenciaram ainda, que à medida que aumentam as porcentagens de cesáreas também aumentam as complicações de saúde para as mães e para os recém-nascidos. Entre os riscos e complicações da cesárea, destacam as hemorragias, as infecções, as reações anestésicas, os problemas respiratórios, dentre outros problemas que prolongam a permanência das parturientes no hospital, podendo levá-las ao óbito. ${ }^{19}$

Assim, no estado do Paraná observase baixa taxa de fecundidade e alta taxa de mortalidade materna. O risco seis vezes mais elevado de morte materna de mulheres 
multigestas (gesta $>5$ ), e a elevada proporção de óbitos considerados evitáveis (90\%) no Paraná apontam para falhas na assistência obstétrica e prováveis lacunas nos serviços de planejamento familiar.

As evidências científicas comprovam que a avaliação do risco obstétrico na primeira consulta de pré-natal, preferencialmente até a $16^{\mathrm{a}}$ semana de gestação, e o encaminhamento oportuno das gestantes de risco para atenção especializada, contribuem para a redução da mortalidade materna, devendo esta ação ser, portanto, implementada em todo o estado, constituindo-se uma rede estadual de atenção ao risco reprodutivo. ${ }^{20}$

As mulheres multigestas neste estudo apresentavam desvantagens sociais e de saú-

\section{REFERÊNCIAS}

1. Say L e Shah I. Mortalidade materna e aborto não-seguro: evitáveis mas persistentes. Boletim Médico da IPPF. Vol. 42-n.2-jun 2008.

2. Toohey JS, Keegan KA, Francis J, Task $\mathrm{S}$, Veciana $\mathrm{M}$, et al. The dangerous multipara: fact or fiction? Am J Obstet Gynecol. 1995 Feb p. 683-5.

3. Bai J, Wong FWS, Bauman A, Mohsin M. Parity and pregnancy outcomes. Am J Gynecol. 2002 Feb; 186(2):274-8.

4. Simonsen SME, Lyon JL, Alder SC, Varner MW. Effect of multiparity on intrapartum and newborn complications in the young women. Obstetrics \& Ginecology. 2005;106:454-60.

5. Aliyu $\mathrm{MH}$ et al. High parity adverse birth outcomes: exploring the maze. Birth. 2005 Mar;32(1):45-59

6. Zimicki S. The relationship between fertility and maternal mortality. In: Parmell AM (org.). Contraceptive use andcontrolled fertility. Washington, D.C.: National Academy Press; 1989. de, que associadas às falhas da assistência obstétrica culminaram na morte precoce das mesmas. Em consequência, pode-se estimar ainda que pelo menos 1000 crianças ficaram órfãs apenas deste grupo de mulheres, criando-se assim mais um grave problema social, uma vez que a maioria das famílias se desestrutura com a ausência materna. ${ }^{22}$

Assim, a identificação e o monitoramento das multigestas com idade avançada e em desvantagem social, ações efetivas de planejamento familiar e serviços obstétricos qualificados para gestação de alto risco poderiam reduzir a mortalidade materna deste grupo de mulheres, diminuindo as taxas do estado como um todo e evitando os problemas sociais delas decorrentes.

7. BRASIL. Ministério da Saúde. Pesquisa Nacional de Demografia e Saúde da Criança e da Mulher. PNDS 2006.Relatório.Brasília/DF;2008.

8. Brasil. Saúde no Brasil 2004 - Uma análise da situação de saúde. Brasília: Ministério da Saúde; 2004.

9. Volochko A. A mensuração da mortalidade materna no Brasil. In: Berquó E (org.). Sexo e Vida Panorama da Saúde Reprodutiva. Campinas: Editora da UNICAMP; 2003.

10. Organización Pan-americana de la Salud (OPS), Organización Mundial de la Salud (OMS). Estratégia regional para a redução da mortalidade e morbidade materna. In: $26^{a}$ Conferência Sanitária Pan-americana, 54 a Sessão do Comitê Regional. Washington; 2003.

11. Soares VMN, Martins, AL. Trajetória e experiência dos Comitês de Prevenção da Mortalidade Materna do Paraná. Rev Bras Mater Infant, Recife. 2006 Oct/ Dec;6(4):453-60.

12. RIPSA - Rede Interagencial de Informações para a Saúde. Indicadores 
básicos para a saúde no Brasil: conceitos e aplicações. Ministério da Saúde.

OPAS. Brasília; 2008.

13. Horta CJG, Fonseca MC. Evolução recente da fecundidade em Minas Gerais. In: IX Seminário sobre a Economia Mineira. Anais do IX Seminário sobre a Economia Mineira, Belo Horizonte, UFMG/CEDEPLAR; 29 de agosto a $1^{\circ}$ de setembro de 2000 .

14. Berquó E e Cavenaghi S. Mapeamento sócio-econômico e demográfico dos regimes de fecundidade no Brasil e sua variação entre 1991 e 2000. In: $14^{\circ}$ Encontro Nacional de Estudos Populacionais, 2004. (Acesso em 22 maio de 2007). Disponível em: http:// www.abep.nepo.unicamp.br

15. Lang CT e King JC. Maternal mortality in the United States. Best Pract Res Clin Obstet Gynaecol; 22(3):517-31, jun 2008.

16. Ramos $\mathrm{S}$ et al. A comprehensive assessment of maternal deaths in Argentina: translating multicentre collaborate research into action. Bulletin of World Health Organization. July 2007, 65(7).
17. Costa AAR et al Mortalidade materna no Recife. Rev Bras Ginecol Obstet.Vol 24, n.2. Rio de Janeiro. Ago 2002

18. Trevisan MR, Lorenzi DRS, Araújo NM, Esber K. Perfil da assistência pré-natal entre usuárias do Sistema Único de Saúde em Caxias do Sul. RBGO. 2002; 24(5).

19. Villar J, Valladares E, Wojdyla D. Caesarean delivery rates and pregnancy outcomes: the 2005 WHO global survey on maternal and perinatal health in Latin

America. Lancet. 2006;367:1819-29.

20. Calderon IMP, Cecatti JG, Vega CEP. Intervenções benéficas no pré-natal para prevenção da mortalidade materna. Rev Bras Ginecol Obstet 2006; 28(5):310-5.

21. Bonan C. e Galli, B. Transformando a cultura da inevitabilidade: a prevenção da mortalidade materna como questão de justiça social. I Fórum Fluminense Maternidade Segura e Cidadania. 2003 http://www.ipas.org.br/arquivos/ opiniao.doc

22. Gomes FA et al. Mortalidade materna na perspectiva do familiar. Rev Esc Enferm USP. 2006; 40(1):50-6.

Recebido em: 20/09/2008

Modificado em: 22/11/2008 Aceito em: 12/12/2008 